\title{
Total $\pi$ Electron Energy of Linear Acenes Nanostructure
}

\author{
Ali Asghar Khakpoor ${ }^{1, a^{*}}$ \\ ${ }^{1}$ Department of Physics, Islamic Azad University- Central Tehran Branch, (IAUCTB), Tehran, Iran \\ aemail: ali.khakpoor@iauctb.ac.ir
}

Keywords: Nanoelectronic; Linear Acenes; Total m Electron Energy; Topological indices.

\begin{abstract}
Moletronics is a branch of nanoelectronic that considers the use of small groups of molecules in nanoscale. A family of organic molecules that has been highly regarded in Moletronics and nano-scale are Acenes with the chemical formula $\mathrm{C}_{4 n+2} \mathrm{H}_{2 n+4}$. Since the identification and analysis of nanostructures, especially in large Acenes need high money and time, a model for predicting the physical and electronic properties is of special importance. Topological indices that were introduced during the studies on the molecular graphs in chemistry can describe and predict some chemical, physical, electronic of the molecules. This paper explains and proves some theorem and then examines topological index F $(\mathrm{G})$ in the linear Acenes family. It is tried to provide an appropriate model to determine the amounts of total $\pi$ electron energy in the family, and especially for the members where the number of loops are high.
\end{abstract}

\section{Introduction}

Nano-electronics is a branch of nanotechnology which invented from the effect of nanotechnology on knowledge and electronics industry. The history of this knowledge dates back to 50 years ago and the time of attempts to reduce the size of transistors as much as possible. Nanoelectronics plays an important role in manufacturing smaller, faster, and more energy-efficient electronic devices. An increase in the amount of the data storage, smaller computer calculators, design of logic circuits, nano-systems, and etc. are among applications of nano-electronics.[1-3]

Nowadays, scientists try to design and manufacture nanometric electronic parts. Manufacturing electronic components in nanoscale involves some restrictions, and it is impossible in most cases. Therefore, the scholars' attention to micro parts in natural scale has recently resulted in the advent of nanostructures in electronics. One of the results of such a link is the design of nano-calculators. Due to possessing p-orbitals and electron cloud above and below them and also the phenomenon of resonance, aromatic hydrocarbons, extracted from benzene, can be a good transmission medium for electrons, and as opposed to chain hydrocarbons, they act like nonconductors. By linking these hydrocarbons, diodes, logical gates, and electronic circuits can be designed.[4][5]

Acenes with the chemical formula $\mathrm{C}_{4 n+2} \mathrm{H}_{2 n+4}$ are a family of organic molecules that are highly considered in molecular electronics and in nanoscale. Based on the evidence, large Acenes with six to ten loops exist in the volcanic ashes and interplanetary dust and are highly suited for the use in electronic components. [6][7]

Since the detection and investigation of the nanostructures family need to spend great money and time, a good model should be existed to predict the electronic properties. One of the cheap and effective methods is to use topological indices.

Topological indices are real numbers that were presented in the studies on the molecular graphs in chemistry and are given in terms of parameters such as degree of vertices, the distance between the vertices, etc. Using these indices, some chemical, physical, and electronic properties of the molecules could be described and predicted.[8-12] Topological indices are divided into different categories according to the definitions. 


\section{Results and Discussion}

A simple graph $G=(V, E)$ is a finite nonempty set $V(G)$ of objects called vertices together with a (possibly empty) set $E(G)$ of unordered pairs of distinct vertices of $G$ called edges. In chemical graphs, the vertices of the graph correspond to the atoms of the molecule, and the edges represent the chemical bonds.A. In chemical graphs, the hydrogen atoms have been removed and ignored and these graphs are termed dehydrogenized. Moreover, all the bonds between atoms are considered as single and the degree of each vertex is a maximum of 4. [13-16]

One of the topological indices is F index which is defined as Eq. 1 [17].

$$
\mathrm{F}=\mathrm{F}(\mathrm{G})=\sum_{v \in V(G)} \operatorname{deg}(v)^{3}=\sum_{u v \in E(G)}\left[\operatorname{deg}(u)^{2}+\operatorname{deg}(v)^{2}\right]
$$

This paper explains and proves some propositions and then examines topological index $F(G)$ given in the relation (1) in the linear Acenes family. It also tried to prove a relation in terms of the number of loops (n) for each index. Then, values of these indices could be obtained in some of the family members of Acenes. Moreover, Gaussian 09 software was used to determine the total $\pi$ electron energy values for the selected members of the family. Finally, it is tried to provide a suitable model for determining the total $\pi$ electron energy values in the family, and especially for the members with high loops. Consider the simple molecular graph of the linear Acenes family $\left(\mathrm{C}_{4 \mathrm{n}+2}\right.$ $\mathrm{H}_{2 \mathrm{n}+4}$ ) as Figure 1 .

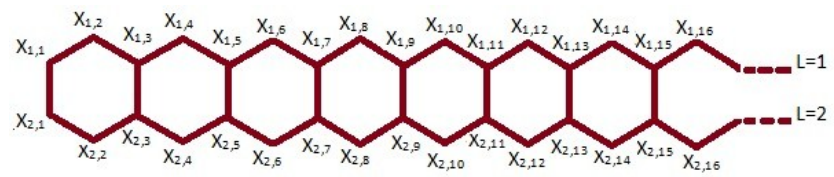

Fig. 1. Molecular graph of the linear Acenes family $\left(\mathrm{C}_{4 n+2} \mathrm{H}_{2 \mathrm{n}+4}\right)$

Theorem: suppose $\mathrm{n}$ is the number of loops in the linear Acenes family. Thus, the index $\mathrm{F}(\mathrm{G})$ is equal to:

$$
F(G)=70 n-22
$$

Proof: consider the simple graph in Fig. 1 that can be partitioned into three areas:

I. All the vertices and edges that are located on and over level $L=1$ are called $G_{1}$, Fig. 2 :

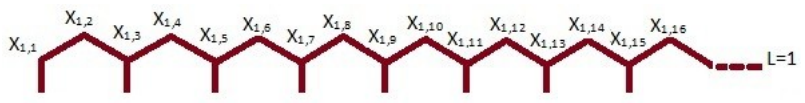

Fig. 2. $\mathrm{G}_{1}$ of all the vertices and edges of the graph in Figure 1 that are located on and over the level $\mathrm{L}=1$.

II. All the vertices and edges that are located on and beneath the level of $\mathrm{L}=2$ are called $\mathrm{G}_{2}$, Fig. 3:

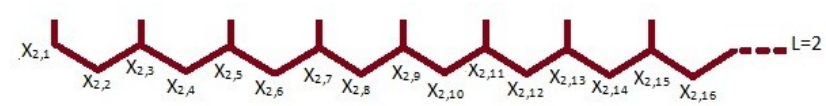

Fig. 3. $G_{2}$ of all the vertices and edges of the graph in Figure 1 that are located on and beneath the level $\mathrm{L}=1$ and $\mathrm{L}=2$. 
III. All the vertices and edges that are located between $\mathrm{L}=1$ and $\mathrm{L}=2$ are called $\mathrm{G}_{3}$, Fig. 4 :

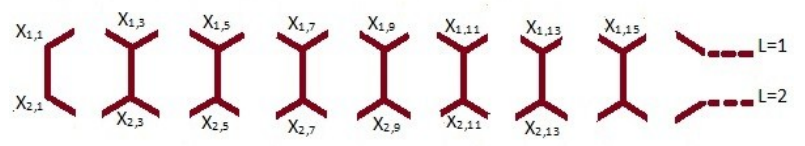

Fig. 4. $\mathrm{G}_{3}$ of all the vertices and edges of the graph in Figure 1 that are located between two levels of $\mathrm{L}=2$ and $\mathrm{L}=1$.

According to Fig. 2 and Eq.1, were having for the $\mathrm{G}_{1}$ area:

$$
F\left(G_{1}\right)=\sum_{m=1}^{k-1}\left[d_{1, m}^{2}+d^{2}{ }_{1, m+1}\right]
$$

For the first and last sentences in the rEq.3, which corresponds to the vertices $X_{1,1}$ and $X_{1, k}$,we have:

$$
d_{1,1}^{2}+d_{1,2}^{2}=d_{1, k-1}^{2}+d_{1, k}^{2}=8
$$

And for $m \neq 1$ and $m \neq k-1$, we have also:

$$
d_{1, m}^{2}+d_{1, m+1}^{2}=13
$$

Thus, according to the Eqs. 3 and 4, Eq. 3 becomes:

$$
F\left(G_{1}\right)=8+\left[\sum_{m=2}^{k-2} 13\right]+8
$$

And since $\mathrm{k}=2 \mathrm{n}+1$, we have:

$$
F\left(G_{1}\right)=16+13[(k-2)-1]=16+26(n-1)
$$

For the $\mathrm{G}_{2}$ area shown in Fig. 3 we have the same result:

$$
F\left(G_{2}\right)=16+26(n-1)
$$

However, according to Figure 4 since only $\mathrm{X}_{1, \mathrm{~m}}$ includes $m \equiv o d d$, we have for the $\mathrm{G}_{3}$ area:

$$
F\left(G_{3}\right)=\mathrm{d}_{1,1}^{2}+\mathrm{d}_{2,1}^{2}+\mathrm{d}_{1, \mathrm{k}}^{2}+\mathrm{d}_{2, \mathrm{k}}^{2}+\sum_{\mathrm{m}=2}^{\frac{\mathrm{k}-1}{2}}\left(\mathrm{~d}_{1, \mathrm{~m}}^{2}+\mathrm{d}_{2, \mathrm{~m}}^{2}\right)
$$

Here, the first and last sentences are separated and we have for these two sentences:

$$
d^{2}{ }_{1,1}+d_{2,1}^{2}=d^{2}{ }_{1, k}+d^{2}{ }_{2, k}=8
$$

In addition, other expressions summed in the relation (9) are equal:

$$
d_{1, m}^{2}+d_{2, m}^{2}=18
$$


Thus, according to the relations (10) and (11), relation (9) is as follows:

$$
F\left(G_{3}\right)=16+18\left[\left(\frac{k-1}{2}\right)-1\right]=16+18(n-1)
$$

And since:

$$
F(G)=\mathrm{F}\left(G_{1}\right)+\mathrm{F}\left(G_{2}\right)+\mathrm{F}\left(G_{3}\right)
$$

And we reach the Eq.2 using the Eqs.7, 8 and 12, the theorem is proved.

\section{Results}

In order to introduce a model that can relate the physical properties of nanostructures to their topological parameters, the first fifteen members of the linear Acenes family $\left(\mathrm{C}_{4 n+2} \mathrm{H}_{2 n+4}\right)$ were selected and $F(G)$ index was calculated by equation (2). The results in are summarized Table 1 . Moreover, the last column of Table 1 shows the calculated values of total electron energy of the selected members of linear Acenes family. Total electron energy family values were calculated by Gaussian 09 software and were compared with the results reported by reliable sources [19].

Table 1. Index F (G) and $E_{\pi}$ in the linear Acenes family

(G)

Fig.5 depicts the changes in $E_{\text {aElectron }}$ of linear Acenes family in terms of the F (G) index. This chart shows the high success of the $\mathrm{F}(\mathrm{G})$ index in anticipation of $E_{\text {aElectron }}$ in linear Acenes family with the accuracy greater than $\mathrm{R}^{2}=0.9978$. Therefore, $E_{\pi \text { Electron }}$ in this family can be predicted in terms of $F(G)$ with the following Equation. 


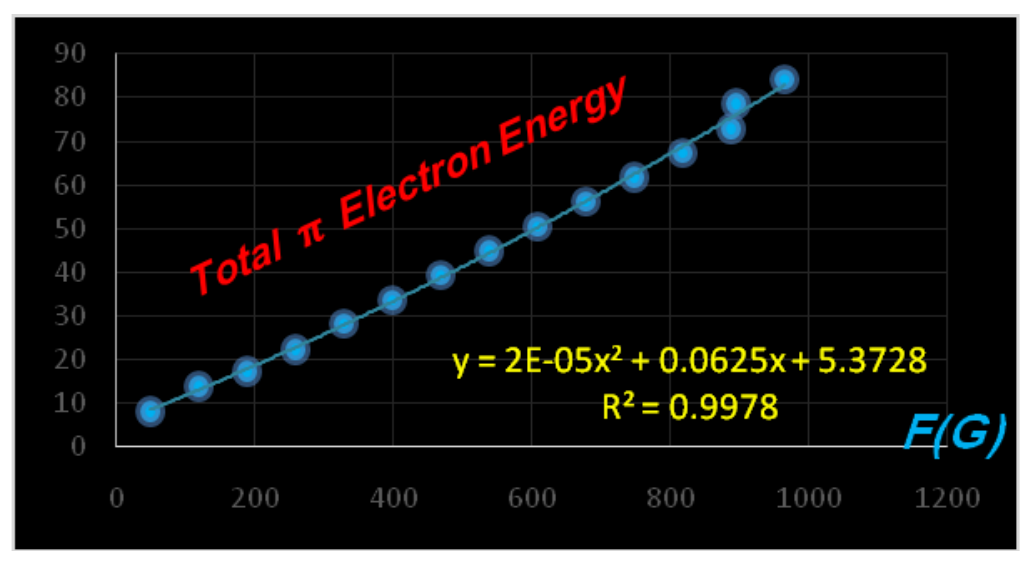

Fig. 5. Changes in $E_{\text {rElectron }}$ of the Linear Acenes family in terms of index $F(G)$

$$
E_{\text {तElectron }}=2 \times 10^{-5} F(G)^{2}+625 \times 10^{-4} F(G)+5.3728
$$

To examine the accuracy of the relation, molecule $\mathrm{C}_{66} \mathrm{H}_{36}$ of the linear Acenes family was selected and the topological index $\mathrm{F}(\mathrm{G})$ for this molecule were calculated using Eq.2. The values are inserted in the Eq.14 and the following value is obtained for $E_{\text {aElectron }}$ of the molecules, Table2.

Table 2. The topological index F (G) and $E_{\text {tElectron }}$ for molecule $\mathrm{C}_{66} \mathrm{H}_{36}$

\begin{tabular}{|c|c|}
\hline Index $\mathrm{F}(\mathrm{G})$ & $E_{\text {rElectron }}(\mathrm{ev})$ \\
\hline 1098 & 98.10988 \\
\hline
\end{tabular}

\section{Conclusions}

In the family of linear Acenes with chemical formula $\mathrm{C}_{4 n+2} \mathrm{H}_{2 n+4}$, it is possible to predict total $\pi$ electron energy using topological index $F(G)$. The predictions that are given by the Eq. 14 have the accuracy greater than $99.58 \%$. According to the proof of theorem lindex F (G) in the family is obtained from Eq.2.

\section{Acknowledgments}

We would like to thank Islamic Azad University Central Tehran Branch (IAUCTB) for helpful protections. Also we acknowledge all those who have helped us with their support and cooperation in conducting this study.

\section{References}

[1]. R. Waser, Nanoelectronics and Information Technology, 3rd Edition, WILEY-VCH 2012.

[2]. F. Patolsky, B.P. Timko, G. Zheng and C.M. Lieber, Nanowire-based nanoelectronic devices in the life sciences, MRS bulletin, Cambridge, Univ Press 2007.

[3]. A.J. Pérez-Jiménez and J.C. Sancho-Garca, Journal of the American Chemical Society, 131 (2009).

[4]. J.C. Sancho-García and A.J. Pérez-Jiménez, Physical Chemistry Chemical Physics 11 (2009) 2741-2746.

[5]. D. Jiang and S. Dai, Chemical Physics Letters 466 (2008) 72-75.

[6]. J. C. Sancho-Garc'ia, and A.J. P'erez-Jim'enez, Phys. Chem. Chem. Phys. 11 (2009) 27412746. 
[7]. J. De-en, and D. Sheng, Chem. Phys. Lett. 466, 1-3 (2008) 72-75.

[8]. A.A. Khakpoor and B. Agahi Keshe, J. of Materials Science and Chemical Engineering 3, 8 (2015) 1-5.

[9]. A. A. Khakpoor, and B. Agahi Keshe, IJCR 7, 5 (2015) 15686-15689.

[10]. A.A. Khakpoor and B. Agahi Keshe, Int. J. of Adv. Res. 3, 5 (2015) 548-551.

[11]. A.A. Khakpoor, Int. J. of Adv. Res., vol. 3, 6 (2015) 1536-1540.

[12]. A.A. Khakpoor and B. Agahi Kesh: submitted to J. of Nanoelectronics and Optoelectronics (2015).

[13]. A.T. Balaban, I. Motoc, D. Bonchev, and O. Mekenyan, Topics Curr. Chem. 114 (1983) 2155.

[14]. R. Todeschini, V. and Consonni, Handbook of Molecular Descriptors, Wiley-VCH, Weinheim 2000 .

[15]. N. Trinajstic, Chemical Graph Theory, 2nd ed., CRC press, Boca Raton, FL, (1992).

[16]. G.S. Ezra, symmetry properties of Molecules. Lecture Notes in chemistry, 28, Springer, Germany 1982.

[17]. B. Furtula and I. Gutman: Accepted to J. Math Chem. 27 January (2015).

DOI 10.1007/s10910-015-0480-z

[18]. L. Turker, Tr. J. of Chemistry. 22 (1998) 109-114. 